\title{
Bottom strange molecules with isospin 0
}

\author{
Zhi-Feng Sun, ${ }^{1, *}$ Ju-Jun Xie, ${ }^{2, \dagger}$ and E. Oset ${ }^{3, \$}$ \\ ${ }^{1}$ School of Physical Science and Technology, Lanzhou University, Lanzhou 730000, China \\ ${ }^{2}$ Institute of Modern Physics, Chinese Academy of Sciences, Lanzhou 730000, China \\ ${ }^{3}$ Departamento de Fsica Terica and IFIC, Centro Mixto Universidad de Valencia-CSIC, \\ Institutos de Investigacíon de Paterna, Aptdo. 22085, 46071 Valencia, Spain
}

(Received 26 January 2018; published 31 May 2018)

\begin{abstract}
Using the local hidden gauge approach, we study the possibility of the existence of bottom strange molecular states with isospin 0 . We find three bound states with spin parity $0^{+}, 1^{+}$, and $2^{+}$generated by the $\bar{K}^{*} B^{*}$ and $\omega B_{s}^{*}$ interaction, among which the state with spin 2 can be identified as $B_{s 2}^{*}(5840)$. In addition, we also study the $\bar{K}^{*} B$ and $\omega B_{s}$ interaction and find a bound state which can be associated to $B_{s 1}(5830)$. In addition, the $\bar{K} B^{*}, \eta B_{s}^{*}, \bar{K} B$, and $\eta B_{s}$ systems are studied, and two bound states are predicted. We expect that further experiments can confirm our predictions.
\end{abstract}

DOI: $10.1103 /$ PhysRevD.97.094031

\section{INTRODUCTION}

The local hidden gauge symmetry was introduced in Refs. [1-4] which regards vector mesons as the gauge bosons and pseudoscalar mesons as the Goldstone bosons. Considering this symmetry together with the global chiral symmetry, one can construct the Lagrangian describing interactions involving vector and pseudoscalar mesons. On the other hand, the Bethe-Salpeter equation is a powerful tool to deal with nonperturbative physics while restoring two-body unitarity in coupled channels. The theory incorporating the above two points has been instrumental in explaining many properties of hadronic resonances. In Ref. [5], the $f_{0}(1370)$ and $f_{2}(1270)$ were explained as resonances generated from the $\rho \rho$ interaction. Later, in Ref. [6] the work of Ref. [5] was extended to SU(3), and five of the generated states were identified with the observed $f_{0}(1370), f_{2}(1270), f_{0}(1710), f_{2}^{\prime}(1525)$, and $K_{2}^{*}(1430)$. In the spin-1 sector, a resonance was also found in Ref. [6] with a mass and width around 1800 and $80 \mathrm{MeV}$, respectively. This state, $h_{1}(1800)$, is dynamically generated from the $K^{*} \bar{K}^{*}$ interaction, and it was investigated in the process $J / \psi \rightarrow \eta K^{* 0} \bar{K}^{* 0}$ in Ref. [7] and in the process $\eta_{c} \rightarrow \phi K^{*} \bar{K}^{*}$ in Ref. [8]. In Ref. [9], the authors studied the interactions of $\rho, \omega$, and $D^{*}$, and three states with spin

\footnotetext{
*sunzf@lzu.edu.cn

†xiejujun@impcas.ac.cn

${ }^{*}$ Eulogio.Oset@ific.uv.es
}

Published by the American Physical Society under the terms of the Creative Commons Attribution 4.0 International license. Further distribution of this work must maintain attribution to the author(s) and the published article's title, journal citation, and DOI. Funded by SCOAP ${ }^{3}$.
$J=0,1,2$ were predicted, among which the second and third ones were identified with $D^{*}(2640)$ and $D_{2}^{*}(2460)$, respectively. The third state predicted, $D(2600)$, was found later in Ref. [10] and has been reconfirmed [11,12]. This work was extended to the case of the $\rho(\omega) B^{*}(B)$ interaction in Ref. [13], where $B_{1}(5721)$ and $B_{2}^{*}(5747)$ were explained as $\rho(\omega) B^{*}$ and $\rho B$ molecules.

The first evidence for at least one of the bottom strange states was found by the OPAL experiment [14]. Evidence for a single state interpreted as $B_{s 2}^{*}$ was seen by the Delphi Collaboration [15]. $B_{s 2}^{*}$ (5840) was observed by both the CDF and D0 collaborations in the $B^{+} K^{-}$channel [16-18]. In the CDF experiment, there is another peak in the $B^{+} K^{-}$ invariant mass spectrum corresponding to $B_{s 1}(5830)$. However, $B_{s 1}(5830) \rightarrow B^{+} K^{-}$is not allowed. The interpretation is that this peak comes from the channel $B^{*+} K^{-}$ and $B^{*+}$ decays to $B^{+} \gamma$ where the photon is not detected. As a consequence, the peak is shifted by the $B^{*}-B$ mass difference due to the missing momentum of the photon. Recently, the LHCb Collaboration first measured the mass and width of $B_{s 2}^{*}(5840)$ in the $B^{*+} K^{-}$channel. In addition, the ratio $\frac{B_{s 2}^{*}(5840) \rightarrow B^{*+} K^{-}}{B_{s 2}^{*}(5840) \rightarrow B^{+} K^{-}}$was measured and the decay of $B_{s 1}(5830) \rightarrow B^{*+} K^{-}$was observed as well [19]. In Refs. [20-22], the $B_{s 0}^{*}$ and $B_{s 1}$ mesons were explained as $B \bar{K}$ and $B^{*} \bar{K}$ molecular states, respectively.

In addition, the D0 Collaboration reported the narrow structure $X(5568)$ in the $B_{s}^{0} \pi^{ \pm}$invariant mass spectrum, whose mass and width are $5567.8 \pm 2.9_{-1.9}^{+0.9}$ and $21.9 \pm 0.6_{-2.5}^{+5.0} \mathrm{MeV}$, respectively [23]. However, the LHCb Collaboration [24], CMS Collaboration [25], and CDF Collaboration [26] claimed that no such decay mode was detected. Recently, the D0 Collaboration made further claims for the $X(5568)$ from the decay $X(5568) \rightarrow B_{s} \pi^{ \pm}$ 
and the result is consistent with the previous measurement by the D0 Collaboration [27]. Within various models, many theoretical groups have studied possible ways to explain $X(5568)$ as a tetraquark state, a molecular state, etc. [28-60] (see also the review [61]). In a previous paper [51] we dealt with this problem, concluding that this state could not be interpreted as a molecular state of $B_{s} \pi, B \bar{K}$ and we do not further discuss this issue here.

In this work, we extrapolate the local hidden gauge approach to the systems containing bottom and strange quarks. The paper is organized as follows. After this Introduction, in Sec. II we show the local hidden gauge Lagrangian from which the potentials are obtained. Then, we construct the $T$ matrix by solving the Bethe-Salpeter equation. In Sec. III we present our results. Finally, we conclude with a short summary.

\section{FORMALISM}

\section{A. Lagrangian}

In order to describe the interaction of bottom and strange mesons, we need to use the local hidden gauge approach, under which vector mesons are treated as gauge bosons. The covariant derivative is defined as

$$
D_{\mu} \xi_{L, R}=\partial_{\mu} \xi_{L, R}-i V_{\mu} \xi_{L, R},
$$

and the gauge field strength as

$$
V_{\mu \nu}=\partial_{\mu} V_{\nu}-\partial_{\nu} V_{\mu}-i g\left[V_{\mu}, V_{\nu}\right] .
$$

Here, $g$ is given by $g=\frac{m_{V}}{2 f_{\pi}}$ with the pion decay constant $f_{\pi}=93 \mathrm{MeV}$, and $m_{V}$ are the masses of vector mesons. $\xi_{L, R}$ are defined as

$$
\begin{aligned}
& \xi_{L}=e^{i \sigma / f_{\sigma}} e^{-i \frac{1}{\sqrt{2}} P / f_{\pi}} \\
& \xi_{R}=e^{i \sigma / f_{\sigma}} e^{i \frac{1}{\sqrt{2}} P / f_{\pi}}
\end{aligned}
$$

In this paper, we take the unitary gauge, i.e., $\sigma=0$. In the above equations, the matrices $V_{\mu}$ and $P$ have the following form:

$$
\begin{aligned}
V_{\mu} & =\left(\begin{array}{cccc}
\frac{\omega}{\sqrt{2}}+\frac{\rho^{0}}{\sqrt{2}} & \rho^{+} & K^{*+} & B^{*+} \\
\rho^{-} & \frac{\omega}{\sqrt{2}}-\frac{\rho^{0}}{\sqrt{2}} & K^{* 0} & B^{* 0} \\
K^{*-} & \bar{K}^{* 0} & \phi & B_{s}^{* 0} \\
B^{*-} & \bar{B}^{* 0} & \bar{B}_{s}^{* 0} & \Upsilon
\end{array}\right)_{\mu} \\
P & =\left(\begin{array}{cccc}
\frac{\eta}{\sqrt{3}}+\frac{\eta^{\prime}}{\sqrt{6}}+\frac{\pi^{0}}{\sqrt{2}} & \pi^{+} & K^{+} & B^{+} \\
\pi^{-} & \frac{\eta}{\sqrt{3}}+\frac{\eta^{\prime}}{\sqrt{6}}-\frac{\pi^{0}}{\sqrt{2}} & K^{0} & B^{0} \\
K^{-} & \bar{K}^{0} & -\frac{\eta}{\sqrt{3}}+\sqrt{\frac{2}{3} \eta^{\prime}} & B_{s}^{0} \\
B^{-} & \bar{B}^{0} & \bar{B}_{s}^{0} & \eta_{b}
\end{array}\right) .
\end{aligned}
$$

After defining the blocks

$$
\begin{gathered}
\hat{\alpha}_{\perp \mu}=\frac{1}{2 i}\left(D_{\mu} \xi_{R} \cdot \xi_{R}^{\dagger}-D_{\mu} \xi_{L} \cdot \xi_{L}^{\dagger}\right), \\
\hat{\alpha}_{\| \mu}=\frac{1}{2 i}\left(D_{\mu} \xi_{R} \cdot \xi_{R}^{\dagger}+D_{\mu} \xi_{L} \cdot \xi_{L}^{\dagger}\right),
\end{gathered}
$$

one can construct the Lagrangian [4]

$$
\mathcal{L}=\mathcal{L}_{A}+a \mathcal{L}_{V}+\mathcal{L}_{I I I},
$$

where

$$
\begin{aligned}
\mathcal{L}_{A} & =f_{\pi}^{2}\left\langle\hat{\alpha}_{\perp \mu} \hat{\alpha}_{\perp}^{\mu}\right\rangle, \\
a \mathcal{L}_{V} & =f_{\sigma}^{2}\left\langle\hat{\alpha}_{\| \mu} \hat{\alpha}_{\|}^{\mu}\right\rangle, \\
\mathcal{L}_{I I I} & =-\frac{1}{4}\left\langle V_{\mu \nu} V^{\mu \nu}\right\rangle,
\end{aligned}
$$

with $f_{\sigma}^{2}=a f_{\pi}^{2}$, and we take $a=2$ as in Ref. [4].

After expanding the Lagrangians in Eq. (7), we get the terms needed in our calculation, i.e., the three-vector vertex

$$
\mathcal{L}_{\mathrm{VVV}}=i g\left\langle\left(\partial_{\mu} V_{\nu}-\partial_{\nu} V_{\mu}\right) V^{\mu} V^{\nu}\right\rangle
$$

four-vector vertex

$$
\mathcal{L}_{\mathrm{VVVV}}=\frac{g^{2}}{2}\left\langle V_{\mu} V_{\nu} V^{\mu} V^{\nu}-V_{\nu} V_{\mu} V^{\mu} V^{\nu}\right\rangle
$$

four-pseudoscalar vertex

$$
\mathcal{L}_{\mathrm{PPPP}}=-\frac{1}{24 f_{\pi}^{2}}\left\langle\left[P, \partial_{\mu} P\right]\left[P, \partial^{\mu} P\right]\right\rangle .
$$

and vector-pseudoscalar-pseudoscalar vertex

$$
\mathcal{L}_{\mathrm{VPP}}=-i g\left\langle V_{\mu}\left[P, \partial^{\mu} P\right]\right\rangle .
$$

Note that there is no vector-vector-pseudoscalarpseudoscalar (VVPP) contact term under the hidden local symmetry. Moreover, since the vector-vector-pseudoscalar interaction is anomalous with a comparatively small contribution, we do not take it into account. In this work, we will study the interaction between bottom and strange mesons, so we extend the $\mathrm{SU}(3)$ flavor symmetry to $\mathrm{SU}(4)$. Next, we change the form of the three-vector Lagrangian in Eq. (9) through some short calculations,

$$
\begin{aligned}
\mathcal{L} & =i g\left\langle\left(\partial_{\mu} V_{\nu}-\partial_{\nu} V_{\mu}\right) V^{\mu} V^{\nu}\right\rangle \\
& =i g\left\langle V_{\mu} V_{\nu} \partial^{\mu} V^{\nu}-V_{\mu} \partial^{\mu} V^{\nu} V_{\nu}\right\rangle \\
& =i g\left\langle V_{\mu}\left[V_{\nu}, \partial^{\mu} V^{\nu}\right]\right\rangle,
\end{aligned}
$$

from which we see that this Lagrangian has a similar form as that in Eq. (12) except for the minus sign. 
As noted in Ref. [62], for small three-momenta of the vector mesons compared to their mass, the $\epsilon^{0}$ component of the external vectors can be neglected. $V_{\mu}$ in the last line of Eq. (13) cannot correspond to an external vector. If this were the case, $\nu$ would be spatial and $\partial^{i}(i=1,2,3)$ would lead to negligible three-momenta of vector mesons. Then, the Lagrangian in Eq. (13) would give zero contribution. Henceforth, we conclude that $V_{\mu}$ in Eq. (13) corresponds to the exchange vector. Similarly, in Eq. (12), $V_{\mu}$ corresponds to the exchanged vector too. So Eqs. (13) and (12) are formally identical, except for an additional factor $\vec{\epsilon} \cdot \vec{\epsilon}$ which comes from $V_{\nu} V^{\nu}$ (note that $\epsilon_{\mu} \epsilon^{\mu}$ gives $-\vec{\epsilon} \cdot \vec{\epsilon}$ ).

It should be noted that the local hidden gauge approach is constructed within $\mathrm{SU}(2)$ or SU(3) $[63,64]$. In the heavyquark sector one cannot invoke heavy mesons as Goldstone bosons. Yet, the extension to the heavy-quark sector is possible because the dominant terms of the interaction correspond to the exchange of light vectors $(\rho, \omega, \phi)$ and the heavy quarks of the hadrons are just spectators. In this case it is possible to make a mapping of the interaction in the heavy-light hadron sector to the one in the heavy hadron sector. For practical purposes one can use the local hidden gauge Lagrangians extrapolated to $\mathrm{SU}(4)$ as in Eq. (5), since for the exchange of light vectors one is only making use of the relevant SU(3) subgroup. Discussions on this issue and the proof of this property can be seen in Sec. II of Ref. [65] and Sec. II and the Appendix of Ref. [66].

\section{B. $\boldsymbol{B}^{*}$ and $\overline{\boldsymbol{K}}^{*}$ interaction}

The interaction terms of $\bar{K}^{*} B^{*}$ and $\omega B_{s}^{*}$ are depicted by the diagrams in Fig. 1, including contact terms and $t$-channel diagrams. Here, we neglect the bottom-meson-exchange diagrams, which have a much smaller contribution due to the heavy mass of bottom mesons. Besides, the amplitude of $\omega B_{s}^{*} \rightarrow \omega B_{s}^{*}$ is zero, because of the Okubo-Zweig-Iizuka (OZI) rule [67-69]. Recalling the isospin doublet $\left(K^{*+}, K^{* 0}\right)$, $\left(\bar{K}^{* 0},-K^{*-}\right),\left(B^{*+}, B^{* 0}\right),\left(\bar{B}^{* 0},-B^{*-}\right)$, and the isospin triplet $\left(-\rho^{+}, \rho^{0}, \rho^{-}\right)$, we have the flavor wave functions

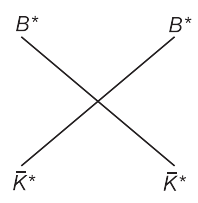

(a)

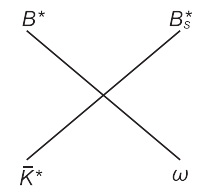

(b)

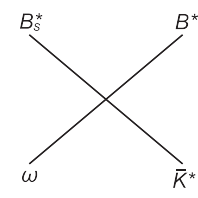

(c)

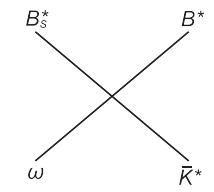

(d)

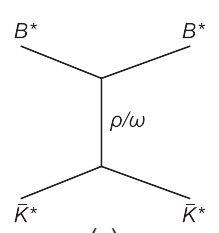

(e)

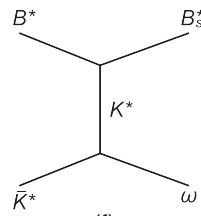

(f)

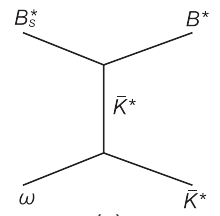

(g)
FIG. 1. Feynman diagrams describing the $\bar{K}^{*} B^{*}$ and $\omega B_{s}^{*}$ interaction. (a), (b), (c) and (d) correspond to contact terms, and (e), (f) and (g) are t-channel diagrams.

$$
\begin{gathered}
\left|\bar{K}^{*} B^{*} ; I=0\right\rangle=\frac{K^{*-} B^{*+}+\bar{K}^{* 0} B^{* 0}}{\sqrt{2}}, \\
\left|\omega B_{s}^{*} ; I=0\right\rangle=\omega B_{s}^{*} .
\end{gathered}
$$

Here the channel $\phi B_{s}^{*}$ is not considered, since its threshold is much higher than those of the other two. With the structure of Eqs. (12) and (13), all of the amplitudes have the structure $\left(k_{1}+k_{3}\right) \cdot\left(k_{2}+k_{4}\right) \epsilon_{\mu 1} \epsilon_{3}^{\mu} \epsilon_{\nu 2} \epsilon_{4}^{\nu}$. As mentioned above, since the three-momenta of the external particles are much smaller than the masses, the $\epsilon^{0}$ component of the external vector mesons can be neglected. So we have $\epsilon_{\mu 1} \epsilon_{\nu 2} \epsilon_{3}^{\mu} \epsilon_{4}^{\nu} \sim \epsilon_{i 1} \epsilon_{j 2} \epsilon_{3}^{i} \epsilon_{4}^{j}, \epsilon_{\mu 1} \epsilon_{2}^{\mu} \epsilon_{\nu 3} \epsilon_{4}^{\nu} \sim \epsilon_{i 1} \epsilon_{2}^{i} \epsilon_{j 3} \epsilon_{4}^{j}, \epsilon_{\mu 1} \epsilon_{\nu 2} \epsilon_{3}^{\nu} \epsilon_{4}^{\mu} \sim$ $\epsilon_{i 1} \epsilon_{j 2} \epsilon_{3}^{j} \epsilon_{4}^{i}$, with $i=1,2$, 3. After writing the amplitudes using Feynman rules, we project the polarization vector products into different spin states:

$$
\begin{gathered}
\mathcal{P}(0)=\frac{1}{3} \epsilon^{i} \epsilon^{i} \epsilon^{j} \epsilon^{j}, \\
\mathcal{P}(1)=\frac{1}{2}\left(\epsilon^{i} \epsilon^{j} \epsilon^{i} \epsilon^{j}-\epsilon^{i} \epsilon^{j} \epsilon^{j} \epsilon^{i}\right), \\
\mathcal{P}(2)=\frac{1}{2}\left(\epsilon^{i} \epsilon^{j} \epsilon^{i} \epsilon^{j}+\epsilon^{i} \epsilon^{j} \epsilon^{j} \epsilon^{i}\right)-\frac{1}{3} \epsilon^{i} \epsilon^{i} \epsilon^{j} \epsilon^{j},
\end{gathered}
$$

where the order of the $\epsilon$ 's is $1,2,3,4$ for the reaction $1+2 \rightarrow 3+4$. Hence, the amplitudes of different spins for $\bar{K}^{*} B^{*} \rightarrow \bar{K}^{*} B^{*}$ (with $\left.I=0\right)$ are

$$
\begin{gathered}
t_{\mathrm{cont}}^{S=0}=4 g^{2}, \\
t_{\mathrm{cont}}^{S=1}=6 g^{2}, \\
t_{\mathrm{cont}}^{S=2}=-2 g^{2}, \\
t_{\mathrm{ex}}^{S=0,1,2}=-\frac{g^{2}}{2}\left(\frac{3}{m_{\rho}^{2}}+\frac{1}{m_{\omega}^{2}}\right)(s-u),
\end{gathered}
$$

and those for $\bar{K}^{*} B^{*} \rightarrow \omega B_{s}^{*}$ are

$$
\begin{gathered}
t_{\mathrm{cont}}^{S=0}=-4 g^{2}, \\
t_{\mathrm{cont}}^{S=1}=0, \\
t_{\mathrm{cont}}^{S=2}=2 g^{2}, \\
t_{\mathrm{ex}}^{S=0,1,2}=\frac{g^{2}}{m_{K^{*}}^{2}}(s-u) .
\end{gathered}
$$

In the above equations, the Mandelstam variables $s$ and $u$ are defined as

$$
s=\left(k_{1}+k_{2}\right)^{2},
$$




$$
u=\left(k_{1}-k_{4}\right)^{2} .
$$

\section{C. $B \overline{\boldsymbol{K}}^{*}$ and $\boldsymbol{B}^{*} \overline{\boldsymbol{K}}$ interactions}

In Fig. 2, we show the diagrams for the $\bar{K}^{*} B$ and $\omega B_{s}$ interaction. Note that under hidden local symmetry, there is no contact term for vector-pseudoscalar scattering. The amplitude of $\omega B_{s} \rightarrow \omega B_{s}$ is zero, because of the OZI rules.

For $\bar{K}^{*} B \rightarrow \bar{K}^{*} B$ with $I=0$ we need the exchange of $\rho$ and $\omega$, and we obtain

$$
t_{\mathrm{ex}}^{S=1}=-\frac{g^{2}}{2}\left(\frac{3}{m_{\rho}^{2}}+\frac{1}{m_{\omega}^{2}}\right)(s-u)
$$

and for $\bar{K}^{*} B \rightarrow \omega B_{s}$

$$
t_{\mathrm{ex}}^{S=1}=\frac{g^{2}}{m_{K^{*}}^{2}}(s-u)
$$

Similarly, we can also get the amplitudes for the $\bar{K} B^{*} \rightarrow$ $\bar{K} B^{*}$ process with $I=0$ as follows:

$$
t_{\mathrm{ex}}^{S=1}=-\frac{g^{2}}{2}\left(\frac{3}{m_{\rho}^{2}}+\frac{1}{m_{\omega}^{2}}\right)(s-u) .
$$

However, according to the diagrams shown in Fig. 3, the calculation for $\bar{K} B^{*} \rightarrow \eta B_{s}^{*}$ with $I=0$ is a little bit different. Using the Feynman rules and considering the flavor wave function, we obtain

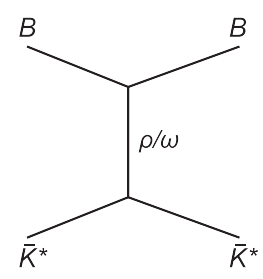

(a)

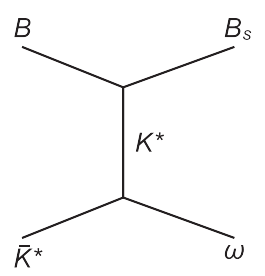

(b)

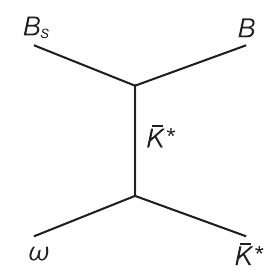

(c)
FIG. 2. Feynman diagrams describing the $\bar{K}^{*} B$ and $\omega B_{s}$ interaction. (a), (b) and (c) correspond to $\rho / \omega, K^{*}$ and $\bar{K}^{*}$ exchange, respectively.

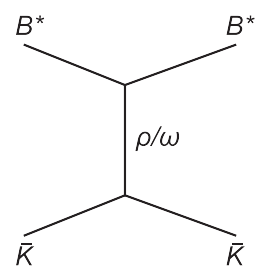

(a)

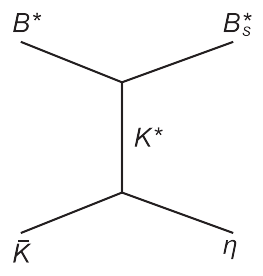

(b)

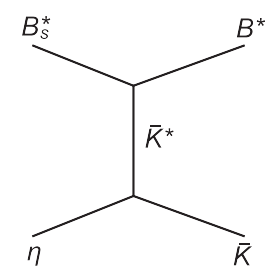

(c)
FIG. 3. Feynman diagrams describing the $\bar{K} B^{*}$ and $\eta B_{s}^{*}$ interaction. (a), (b) and (c) correspond to $\rho / \omega, K^{*}$ and $\bar{K}^{*}$ exchange, respectively.

$$
t_{\mathrm{ex}}^{S=1}=-\frac{2 \sqrt{6} g^{2}}{3 m_{K^{*}}^{2}}(s-u)
$$

\section{D. $B$ and $\bar{K}$ interaction}

In Fig. 4, we show the diagrams depicting the interaction of pseudoscalar and pseudoscalar mesons. The amplitude of the contact terms corresponding to Eq. (11) for the $\bar{K} B \rightarrow \bar{K} B$ process with $I=0$ are

$$
t_{\mathrm{cont}}^{S=0}=-\frac{1}{6 f^{2}}(2 u-t-s),
$$

those for the $\bar{K} B \rightarrow \eta B_{s}$ process are

$$
t_{\mathrm{cont}}^{S=0}=-\frac{\sqrt{6}}{12 f^{2}}(s-u)
$$

and those for the $\eta B_{s} \rightarrow \eta B_{s}$ process are

$$
t_{\mathrm{cont}}^{S=0}=-\frac{1}{36 f^{2}}(-2 t+u+s),
$$

with $t=\left(k_{1}-k_{3}\right)^{2}$. The amplitudes of $t$-channel diagrams for $\bar{K} B \rightarrow \bar{K} B$ are

$$
t_{\mathrm{ex}}^{S=0}=-\frac{g^{2}}{2}\left(\frac{3}{m_{\rho}^{2}}+\frac{1}{m_{\omega}^{2}}\right)(s-u),
$$

and those for $\bar{K} B \rightarrow \eta B_{s}$ are

$$
t_{\mathrm{ex}}^{S=1}=-\frac{2 \sqrt{6} g^{2}}{3 m_{K^{*}}^{2}}(s-u) .
$$

The $t$-channel diagrams for $\eta B_{s} \rightarrow \eta B_{s}$ give zero contribution.

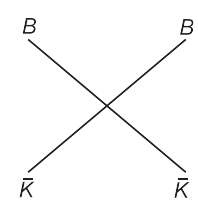

(a) (b)

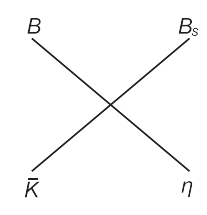

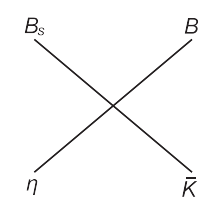

(c)

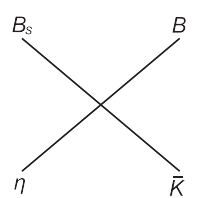

(d)

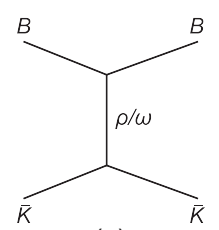

(e)

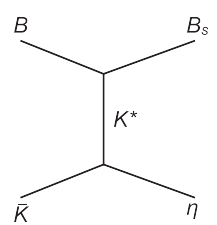

(f)

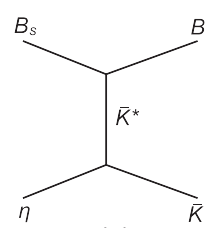

(g)
FIG. 4. Feynman diagrams describing the $\bar{K} B$ and $\eta B_{s}$ interaction. (a), (b), (c) and (d) correspond to contact terms, and (e), (f) and (g) are t-channel diagrams. 


\section{E. $T$ matrix}

With the preparation above, using the Bethe-Salpeter equation in its on-shell factorized form, we obtain the $T$ matrix

$$
T=(I-V G)^{-1} V,
$$

where $V$ corresponds to the transition amplitudes shown above, but projected to the $s$ wave. So we neglect the product $\vec{k}_{1} \cdot \vec{k}_{3}$ in the Mandelstam variables $u$ and $t$ which corresponds to the $p$-wave contribution, i.e.,

$$
\begin{aligned}
u & \approx \frac{m_{1}^{2}+m_{2}^{2}+m_{3}^{2}+m_{4}^{2}}{2}-\frac{\left(m_{4}^{2}-m_{3}^{2}\right)\left(m_{1}^{2}-m_{2}^{2}\right)}{2 s}, \\
t & \approx \frac{m_{1}^{2}+m_{2}^{2}+m_{3}^{2}+m_{4}^{2}}{2}+\frac{\left(m_{4}^{2}-m_{3}^{2}\right)\left(m_{1}^{2}-m_{2}^{2}\right)}{2 s} .
\end{aligned}
$$

$G$ is the two-meson loop function

$G=i \int \frac{d^{4} q}{(2 \pi)^{4}} \frac{1}{q^{2}-m_{1}^{2}+i \epsilon} \frac{1}{(P-q)^{2}-m_{2}^{2}+i \epsilon}$.

Using a cutoff for the three-momentum, we have

$$
G=\int_{0}^{q_{\max }} \frac{q^{2} d q}{(2 \pi)^{2}} \frac{\omega_{1}+\omega_{2}}{\omega_{1} \omega_{2}\left[\left(P^{0}\right)^{2}-\left(\omega_{1}+\omega_{2}\right)^{2}+i \epsilon\right]} .
$$

This integral was already done (see Ref. [70]), and we show it as follows:

$$
\begin{aligned}
G= & \frac{1}{32 \pi^{2}}\left[\frac{\nu}{s}\left\{\log \frac{s-\Delta+\nu \sqrt{1+\frac{m_{1}^{2}}{q_{\max }^{2}}}}{-s+\Delta+\nu \sqrt{1+\frac{m_{1}^{2}}{q_{\max }^{2}}}}+\log \frac{s+\Delta+\nu \sqrt{1+\frac{m_{1}^{2}}{q_{\max }^{2}}}}{-s-\Delta+\nu \sqrt{1+\frac{m_{1}^{2}}{q_{\max }^{2}}}}\right\}-\frac{\Delta}{s} \log \frac{m_{1}^{2}}{m_{2}^{2}}\right. \\
& \left.+2 \frac{\Delta}{s} \log \frac{1+\sqrt{1+\frac{m_{1}^{2}}{q_{\max }^{2}}}}{1+\sqrt{1+\frac{m_{2}^{2}}{q_{\max }^{2}}}}+\log \frac{m_{1}^{2} m_{2}^{2}}{q_{\max }^{2}}-2 \log \left[\left(1+\sqrt{1+\frac{m_{1}^{2}}{q_{\max }^{2}}}\right)\left(1+\sqrt{1+\frac{m_{2}^{2}}{q_{\max }^{2}}}\right)\right]\right] .
\end{aligned}
$$

In Eqs. (40)-(42), $P$ is the total four-momentum of the two mesons in the loop, $m_{1}$ and $m_{2}$ are the masses, $q_{\max }$ stands for the cutoff, $\omega_{i}=\sqrt{\vec{q}_{i}^{2}+m_{i}^{2}}, P^{0}$ is nothing but the center-of-mass energy $\sqrt{s}, \Delta=m_{2}^{2}-m_{1}^{2}$, and $\nu=\sqrt{\left[s-\left(m_{1}+m_{2}\right)^{2}\right]\left[s-\left(m_{1}-m_{2}\right)^{2}\right]}$.

\section{RESULTS}

\section{A. Discussion of the couplings under SU(4) symmetry}

In this subsection, we follow Refs. [13,65,71] and discuss the couplings in the Lagrangian. As an example, we consider the vertex of $B^{*} B^{*} \rho$. In order to estimate the corresponding coupling, we need to compare this vertex with that of $K^{*} K^{*} \rho$, since their topology is the same if the $\bar{s}$

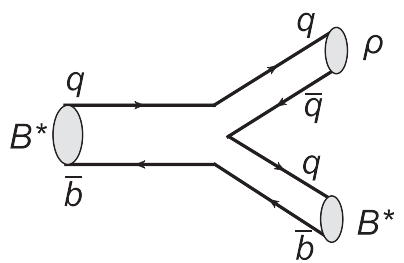

(a)

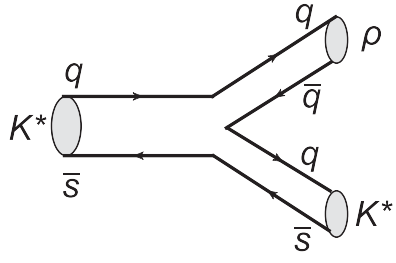

(b)
FIG. 5. The vertexes of $B^{*} B^{*} \rho$ and $K^{*} K^{*} \rho$ at the quark level, which correspond to (a) and (b), respectively. and $\bar{b}$ quarks are seen as spectators. Figure 5 shows the diagrams for these two vertices at the quark level, in which case the corresponding $S$ matrices should be the same, i.e.,

$S^{\text {mic }}=1-i t \sqrt{\frac{2 m_{L}}{2 E_{L}}} \sqrt{\frac{2 m_{L}^{\prime}}{2 E_{L}^{\prime}}} \sqrt{\frac{1}{2 \omega_{\rho}}} \frac{1}{\mathcal{V}^{3 / 2}}(2 \pi)^{4} \delta\left(P_{\text {in }}-P_{\text {out }}\right)$.

On the other hand, at the hadronic level, the $S$ matrices are written as

$S_{B^{*}}^{\mathrm{mac}}=1-i t_{B^{*}} \frac{1}{\sqrt{2 \omega_{B^{*}}}} \frac{1}{\sqrt{2 \omega_{B^{*}}}} \frac{1}{\sqrt{2 \omega_{\rho}}} \frac{1}{\mathcal{V}^{3 / 2}}(2 \pi)^{4} \delta\left(P_{\text {in }}-P_{\text {out }}\right)$,

$S_{K^{*}}^{\mathrm{mac}}=1-i t_{K^{*}} \frac{1}{\sqrt{2 \omega_{K^{*}}}} \frac{1}{\sqrt{2 \omega_{K^{*}}}} \frac{1}{\sqrt{2 \omega_{\rho}}} \frac{1}{\mathcal{V}^{3 / 2}}(2 \pi)^{4} \delta\left(P_{\text {in }}-P_{\text {out }}\right)$.

As discussed above, we should have $S_{B^{*}}^{\mathrm{mac}}=S_{K^{*}}^{\mathrm{mac}}$ which tells us that the corresponding $T$ matrices obey the following relation at the threshold: 


$$
\frac{t_{B^{*}}}{t_{K^{*}}}=\frac{m_{B^{*}}}{m_{K^{*}}} .
$$

If we use the Lagrangian in Eq. (9) and calculate the $T$ matrices of the processes in Fig. 5, we find that Eq. (46) holds automatically when the $\rho$ is the exchanged (virtual) vector meson, because the amplitude has the $\partial^{\mu} \cong \partial^{0}$ operator acting on the external vectors. The coupling of $B^{*} B^{*} \rho$ in Eq. (9) correctly implements the field correction factor of Eq. (46). Since in this case the $b$ quark acts as a spectator in the vertex, this amplitude is automatically consistent with heavy-quark spin symmetry [72]. Similar discussions can be applied to the $B B \rho$ vertex with respect to $K K \rho$, and we have

$$
\frac{t_{B}}{t_{K}}=\frac{m_{B}}{m_{K}}
$$

but this is what we obtain from Eq. (12) using SU(4) flavor symmetry. Effectively one is using SU(3) when the heavy quark is considered as a spectator. In summary, we apply the Lagrangians of Sec. II A, and this automatically takes into account all the elements discussed above.

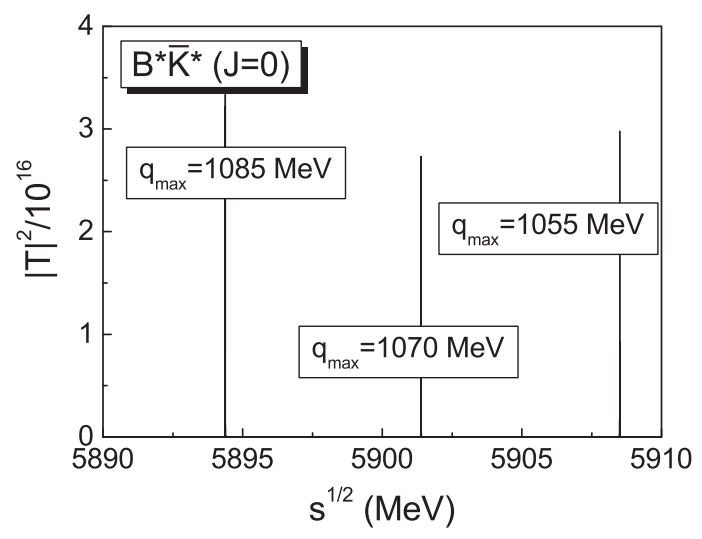

\section{B. The $\overline{\boldsymbol{K}}^{*} \boldsymbol{B}^{*}$ system}

With the potentials given in the previous section, we solve the Bethe-Salpeter equation considering the $\bar{K}^{*} B^{*}$, $\omega B_{s}^{*}$, and $\phi B_{s}^{*}$ coupled channels. We also obtain three bound states with $J=0,1,2$, using the cutoff $q_{\max }$ around $1055-1085 \mathrm{MeV}$. The obtained mass is $5847.8-$ $5831.7 \mathrm{MeV}$ for the spin-2 state which is consistent with that of $B_{s 2}^{*}(5840)$. With this $q_{\max }$, we predict that the bound state with $J=0$ has a mass of $5908.5-5894.4 \mathrm{MeV}$, and the one with $J=1$ has a mass of $5912.1-5898.2 \mathrm{MeV}$. In Fig. 6, we plot the line shape of the mass distribution of these three states. The Particle Data Group (PDG) [73] reports that the mass of $B_{s 1}(5830)$ with spin 1 is smaller than that of $B_{s 2}^{*}(5840)$. However, the generated bound state with spin 1 has a mass about $65 \mathrm{MeV}$ larger than that of the bound state with spin 2. Henceforth, it is difficult to explain the $B_{s 1}(5830)$ as the $\bar{K}^{*} B^{*}$ bound state. In the next subsection, we will come back to this problem.

The $T$ matrix close to a pole behaves like

$$
T_{i j} \approx \frac{g_{i} g_{j}}{z-z_{R}}
$$

where $i, j=\bar{K}^{*} B^{*}, \omega B_{s}^{*}, \phi B_{s}^{*}, g_{i}$ is the coupling to the channel $i, \operatorname{Re}\left(z_{R}\right)$ gives the mass of the bound state, $\operatorname{Im}\left(z_{R}\right)$

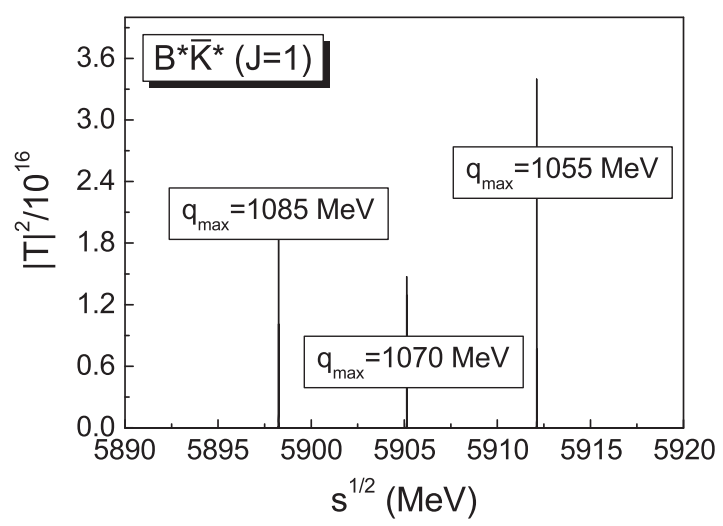

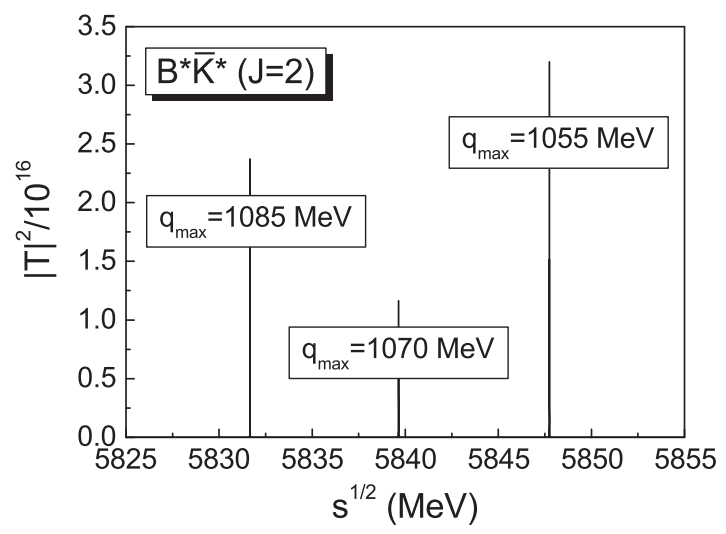

FIG. 6. Squared amplitude for $\bar{K}^{*} B^{*} / \omega B_{s}^{*} / \phi B_{s}^{*}$ systems with spin 0,1 , and 2 , respectively. 
TABLE I. The couplings for $\bar{K}^{*} B^{*}$ systems mixing with $\omega B_{s}^{*}$, $\phi B_{s}^{*}$ channels. Here we chose the typical value of the cutoff as $1070 \mathrm{MeV}$. All values are given in units of MeV.

\begin{tabular}{lrrr}
\hline \hline Channel & $J=0$ & $J=1$ & $J=2$ \\
\hline $\bar{K}^{*} B^{*}$ & 45955 & 45070 & 49633 \\
$\omega B_{s}^{*}$ & -10696 & -14810 & -15017 \\
$\phi B_{s}^{*}$ & 18614 & 15702 & 19409 \\
\hline \hline
\end{tabular}

gives the half width, and $z$ is the complex value of the Mandelstam variable $s$. The coupling for a certain channel is obtained as

$$
g_{i}^{2}=\lim _{z \rightarrow z_{R}} T_{i i}\left(z-z_{R}\right)
$$

The sign of the coupling to the $B^{*} \bar{K}^{*}$ channel is chosen as positive, and those for the other channels are then determined by the following formula:

$$
\frac{g_{i}}{g_{j}}=\lim _{z \rightarrow z_{R}} \frac{T_{i i}}{T_{i j}}
$$

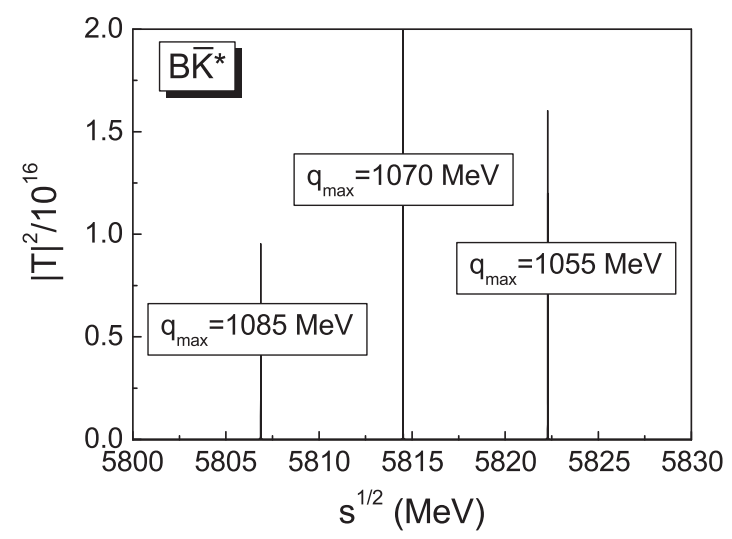

FIG. 7. Squared amplitude for the $\bar{K}^{*} B / \omega B_{s} / \phi B_{s}$ sector depending on the center-of-mass energy.

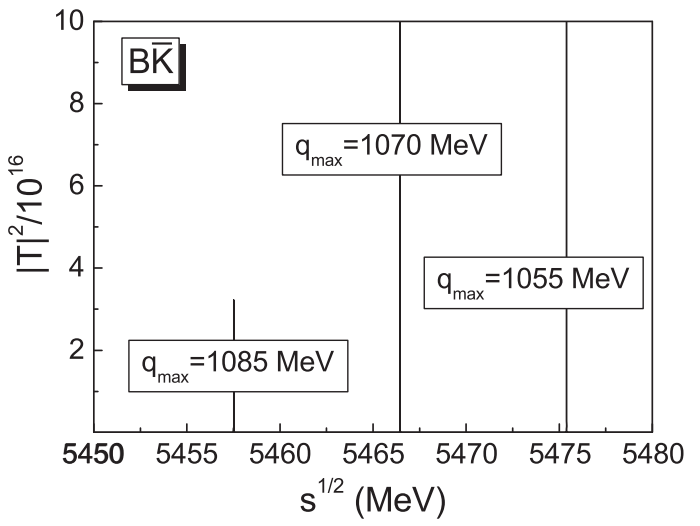

The value of the couplings are listed in Table I, from which we can see that the $\bar{K}^{*} B^{*}$ component is dominant for all of the states.

\section{The $\overline{\boldsymbol{K}}^{*} \boldsymbol{B}$ system}

As mentioned in the previous subsection, the $B_{s 1}(5830)$ cannot be explained as the $\bar{K}^{*} B^{*}$ bound state with spin 1 , since in the PDG the mass of $B_{s 1}(5830)$ is smaller than that of $B_{s 2}^{*}(5840)$, which is contrary to our results. Now we will try to explain the $B_{s 1}(5830)$ under the $\bar{K}^{*} B / \omega B_{s}$ system.

Under hidden local symmetry there are no contact terms for the VVPP vertex, so only vector-exchange diagrams are involved. For the vector-exchange terms, the interactions we study in this subsection have the same form as that of the $\bar{K}^{*} B / \omega B_{s} / \phi B_{s}$ interactions. So here we expect to find a bound state like in the case of the $\bar{K}^{*} B^{*}$ system. We use $q_{\max }=1055-1085 \mathrm{MeV}$ fixed in the case of the $\bar{K}^{*} B^{*}$ bound state with spin 2 . Then we obtain a pole position in the range 5822.3-5806.9 MeV, which is consistent with the mass of $B_{s 1}(5830)$ in the PDG. In Fig. 7, we plot the line shape of $|T|^{2}$ depending on the center-of-mass energy $\sqrt{s}$. We also calculate the couplings, which have the values $g_{\bar{K}^{*} B}=47654, g_{\omega B_{s}}=-13388$, and $g_{\phi B_{s}}=18855$ with the cutoff $q_{\max }=1070 \mathrm{MeV}$.

\section{Other predictions}

In this subsection, we will show the results corresponding to $\bar{K} B^{*} / \eta B_{s}^{*}$ and $\bar{K} B / \eta B_{s}$ interactions.

Like the case of the $\bar{K}^{*} B / \omega B_{s} / \phi B_{s}$ system, there are no contact terms for the $\bar{K} B^{*} / \eta B_{s}^{*}$ interaction. Only the vectormeson exchange diagrams are considered. In Fig. 8, we plot the squared amplitude depending on the center-of-mass energy $\sqrt{s}$. Here, we also use the cutoff $q_{\max }=$ $1055-1085 \mathrm{MeV}$ as before. The pole position is located at $5671.2-5663.6 \mathrm{MeV}$. The couplings of $B^{*} \bar{K}$ and $B_{s}^{*} \eta$ are 30637 and $-13919 \mathrm{MeV}$, respectively, where we choose the cutoff as $1070 \mathrm{MeV}$.

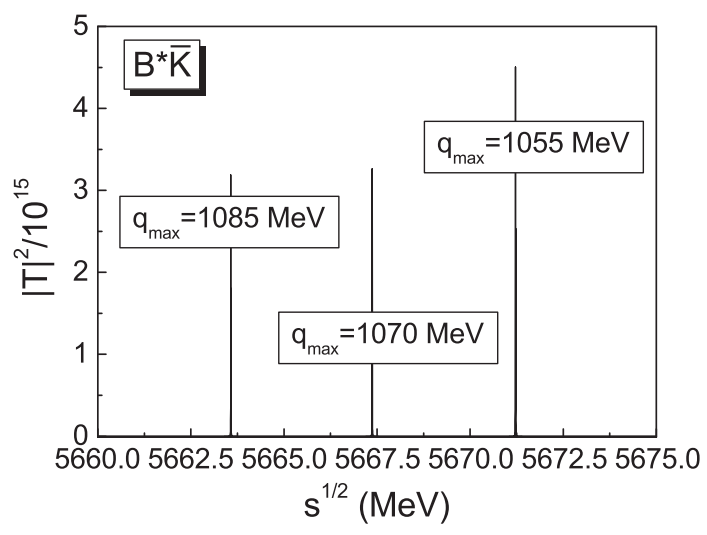

FIG. 8. Squared amplitude for the $\bar{K} B / \eta B_{s}$ and $\bar{K} B^{*} / \eta B_{s}^{*}$ sector. 
TABLE II. Summary of our results where the cutoff is in the range 1055-1085 MeV. Masses in this table are in units of MeV.

\begin{tabular}{llcccccc}
\hline \hline State mass & $I\left(J^{p}\right)$ & Main component & Experiment & State mass & $I\left(J^{p}\right)$ & Main component & Experiment \\
\hline $5475.4-5457.5$ & $0\left(0^{+}\right)$ & $\bar{K} B$ & $\ldots$ & $5908.5-5894.4$ & $0\left(0^{+}\right)$ & $\bar{K}^{*} B^{*}$ & $\ldots$ \\
$5671.2-5663.6$ & $0\left(1^{+}\right)$ & $\bar{K} B^{*}$ & $\ldots$ & $5912.1-5898.2$ & $0\left(1^{+}\right)$ & $\bar{K}^{*} B^{*}$ & $\ldots$ \\
$5822.3-5806.9$ & $0\left(1^{+}\right)$ & $\bar{K}^{*} B$ & $B_{s 1}(5830)$ & $5847.8-5831.7$ & $0\left(2^{+}\right)$ & $\bar{K}^{*} B^{*}$ & $B_{s 2}^{*}(5840)$ \\
\hline \hline
\end{tabular}

For the $\bar{K} B / \eta B_{s}$ system, we predict a bound state with a mass of 5475.4-5457.5 MeV, and the couplings $g_{\bar{K} B}=$ $53577 \mathrm{MeV}$ and $g_{\eta B_{s}}=-3689 \mathrm{MeV}$, with a cutoff $q_{\max }=1070 \mathrm{MeV}$.

We list our results for all of the systems in Table II.

\section{SUMMARY}

In this work, we have studied the systems containing bottom and strange quarks using the chiral unitary approach. Considering $\bar{K}^{*} B^{*}$ and $\omega B_{s}^{*}$ coupled channels and solving the Bethe-Salpeter equation, we found three states with masses 5908.5-5894.4, 5912.1-5898.2, and $5847.8-5831.7 \mathrm{MeV}$, with the cutoff $q_{\max }$ chosen as $1055-$ $1085 \mathrm{MeV}$. The state with spin 2 can be identified with $B_{s 2}^{*}(5840)$. From the couplings that we obtained, we can see that the $\bar{K}^{*} B^{*}$ component is dominant. However, the $B_{s 1}(5830)$ cannot be explained as the state with spin 1, since its mass is smaller than that of $B_{s 2}^{*}(5840)$. So we studied another system, i.e., the $\bar{K}^{*} B / \omega B_{s}$ system, and we found a bound state with a mass $5822.3-5806.9 \mathrm{MeV}$ which agrees with the mass of $B_{s 1}(5830)$. In addition, we also studied $\bar{K} B^{*} / \eta B_{s}^{*}$ and $\bar{K} B / \eta B_{s}$ interactions, and predicted two bound states with masses 5671.2-5663.6 and $5475.4-5457.5 \mathrm{MeV}$, respectively. We expect further experiments to confirm our predictions.

\section{ACKNOWLEDGMENTS}

This work is partly supported by the National Science Foundation for Young Scientists of China under Grants No. 11705069 and the Fundamental Research Funds for the Central Universities. It is partly supported by the National Natural Science Foundation of China (Grants No. 11475227 and No. 11735003) and the Youth Innovation Promotion Association CAS (No. 2016367). This work is also partly supported by the Spanish Ministerio de Economia y Competitividad and European FEDER funds under the Contract No. FIS2011-28853C02-01, No. FIS2011- 28853-C02-02, No. FIS201457026-REDT, No. FIS2014-51948-C2- 1-P, and No. FIS2014-51948-C2-2-P, and the Generalitat Valenciana in the program Prometeo II-2014/068.
[1] M. Bando, T. Kugo, S. Uehara, K. Yamawaki, and T. Yanagida, Phys. Rev. Lett. 54, 1215 (1985).

[2] M. Bando, T. Kugo, and K. Yamawaki, Phys. Rep. 164, 217 (1988).

[3] U. G. Meissner, Phys. Rep. 161, 213 (1988).

[4] M. Harada and K. Yamawaki, Phys. Rep. 381, 1 (2003).

[5] R. Molina, D. Nicmorus, and E. Oset, Phys. Rev. D 78, 114018 (2008).

[6] L. S. Geng and E. Oset, Phys. Rev. D 79, 074009 (2009).

[7] J. J. Xie, M. Albaladejo, and E. Oset, Phys. Lett. B 728, 319 (2014).

[8] X. L. Ren, L. S. Geng, E. Oset, and J. Meng, Eur. Phys. J. A 50, 133 (2014).

[9] R. Molina, H. Nagahiro, A. Hosaka, and E. Oset, Phys. Rev. D 80, 014025 (2009).

[10] P. del Amo Sanchez et al. (BABAR Collaboration), Phys. Rev. D 82, 111101 (2010).

[11] R. Aaij et al. (LHCb Collaboration), J. High Energy Phys. 09 (2013) 145.

[12] R. Aaij et al. (LHCb Collaboration), Phys. Rev. D 94, 072001 (2016).
[13] P. Fernandez-Soler, Z. F. Sun, J. Nieves, and E. Oset, Eur. Phys. J. C 76, 82 (2016).

[14] R. Akers et al. (OPAL Collaboration), Z. Phys. C 66, 19 (1995).

[15] M. Moch (DELPHI Collaboration), Proc. Sci., HEP2005 (2006) 232.

[16] R. K. Mommsen, Nucl. Phys. B, Proc. Suppl. 170, 172 (2007).

[17] T. Aaltonen et al. (CDF Collaboration), Phys. Rev. Lett. 100, 082001 (2008).

[18] V. M. Abazov et al. (D0 Collaboration), Phys. Rev. Lett. 100, 082002 (2008).

[19] R. Aaij et al. (LHCb Collaboration), Phys. Rev. Lett. 110, 151803 (2013).

[20] F. K. Guo, P. N. Shen, H. C. Chiang, R. G. Ping, and B. S. Zou, Phys. Lett. B 641, 278 (2006).

[21] F. K. Guo, P. N. Shen, and H. C. Chiang, Phys. Lett. B 647, 133 (2007).

[22] A. Faessler, T. Gutsche, V. E. Lyubovitskij, and Y. L. Ma, Phys. Rev. D 77, 114013 (2008).

[23] V. M. Abazov et al. (D0 Collaboration), Phys. Rev. Lett. 117, 022003 (2016). 
[24] R. Aaij et al. (LHCb Collaboration), Phys. Rev. Lett. 117, 152003 (2016); 118, 109904(E) (2017).

[25] A. M. Sirunyan et al. (CMS Collaboration), arXiv: 1712.06144.

[26] T. A. Aaltonen et al. (CDF Collaboration), arXiv: 1712.09620.

[27] V. M. Abazov et al. (D0 Collaboration), arXiv:1712.10176.

[28] W. Chen, H. X. Chen, X. Liu, T. G. Steele, and S. L. Zhu, Phys. Rev. Lett. 117, 022002 (2016).

[29] S. S. Agaev, K. Azizi, and H. Sundu, Phys. Rev. D 93, 074024 (2016).

[30] C. M. Zanetti, M. Nielsen, and K. P. Khemchandani, Phys. Rev. D 93, 096011 (2016).

[31] Z. G. Wang, Commun. Theor. Phys. 66, 335 (2016).

[32] L. Tang and C. F. Qiao, Eur. Phys. J. C 76, 558 (2016).

[33] S. S. Agaev, K. Azizi, and H. Sundu, Eur. Phys. J. Plus 131, 351 (2016).

[34] S. S. Agaev, K. Azizi, and H. Sundu, Phys. Rev. D 93, 114007 (2016).

[35] Z. G. Wang, Eur. Phys. J. C 76, 279 (2016).

[36] J. M. Dias, K. P. Khemchandani, A. Martnez Torres, M. Nielsen, and C. M. Zanetti, Phys. Lett. B 758, 235 (2016).

[37] S. S. Agaev, K. Azizi, and H. Sundu, Phys. Rev. D 93, 114036 (2016).

[38] S. S. Agaev, K. Azizi, and H. Sundu, Phys. Rev. D 93, 094006 (2016).

[39] Y. R. Liu, X. Liu, and S. L. Zhu, Phys. Rev. D 93, 074023 (2016).

[40] F. Stancu, J. Phys. G 43, 105001 (2016).

[41] W. Wang and R. Zhu, Chin. Phys. C 40, 093101 (2016).

[42] A. Ali, L. Maiani, A. D. Polosa, and V. Riquer, Phys. Rev. D 94, 034036 (2016).

[43] X. H. Liu and G. Li, Eur. Phys. J. C 76, 455 (2016).

[44] X. W. Kang and J. A. Oller, Phys. Rev. D 94, 054010 (2016).

[45] Y. Jin, S. Y. Li, and S. Q. Li, Phys. Rev. D 94, 014023 (2016).

[46] X. G. He and P. Ko, Phys. Lett. B 761, 92 (2016).

[47] T. J. Burns and E. S. Swanson, Phys. Lett. B 760, 627 (2016).

[48] R. Chen and X. Liu, Phys. Rev. D 94, 034006 (2016).

[49] F. K. Guo, U. G. Meißner, and B. S. Zou, Commun. Theor. Phys. 65, 593 (2016).
[50] Q. F. Lü and Y. B. Dong, Phys. Rev. D 94, 094041 (2016).

[51] M. Albaladejo, J. Nieves, E. Oset, Z. F. Sun, and X. Liu, Phys. Lett. B 757, 515 (2016).

[52] X. Chen and J. Ping, Eur. Phys. J. C 76, 351 (2016).

[53] C. B. Lang, D. Mohler, and S. Prelovsek, Phys. Rev. D 94, 074509 (2016).

[54] R. Albuquerque, S. Narison, A. Rabemananjara, and D. Rabetiarivony, Int. J. Mod. Phys. A 31, 1650093 (2016).

[55] J. X. Lu, X. L. Ren, and L. S. Geng, Eur. Phys. J. C 77, 94 (2017).

[56] B. X. Sun, F. Y. Dong, and J. L. Pang, Chin. Phys. C 41, 074104 (2017).

[57] S. S. Agaev, K. Azizi, B. Barsbay, and H. Sundu, Eur. Phys. J. A 53, 11 (2017).

[58] Z. Yang, Q. Wang, and U. G. Meißner, Phys. Lett. B 767, 470 (2017).

[59] J. R. Zhang, J. L. Zou, and J. Y. Wu, Chin. Phys. C 42, 043101 (2018).

[60] H. W. Ke and X. Q. Li, Eur. Phys. J. C 78, 364 (2018).

[61] H. X. Chen, W. Chen, X. Liu, Y. R. Liu, and S. L. Zhu, Rep. Prog. Phys. 80, 076201 (2017).

[62] E. Oset and A. Ramos, Eur. Phys. J. A 44, 445 (2010).

[63] G. Ecker, J. Gasser, H. Leutwyler, A. Pich, and E. de Rafael, Phys. Lett. B 223, 425 (1989).

[64] H. Nagahiro, L. Roca, A. Hosaka, and E. Oset, Phys. Rev. D 79, 014015 (2009).

[65] S. Sakai, L. Roca, and E. Oset, Phys. Rev. D 96, 054023 (2017).

[66] W. H. Liang, J. M. Dias, V. R. Debastiani, and E. Oset, Nucl. Phys. B930, 524 (2018).

[67] S. Okubo, Phys. Lett. 5, 165 (1963).

[68] G. Zweig, in Developments in the Quark Theory of Hadrons, Vol. 1, edited by D. Lichtenberg and S. Rosen (Hadronic Press, Nonantum, MA, 1980), p. 22.

[69] J. Iizuka, Prog. Theor. Phys. Suppl. 37, 21 (1966).

[70] J. A. Oller, E. Oset, and J. R. Pelaez, Phys. Rev. D 59, 074001 (1999); 60, 099906(E) (1999); 75, 099903(E) (2007).

[71] W. H. Liang, C. W. Xiao, and E. Oset, Phys. Rev. D 89, 054023 (2014).

[72] A. V. Manohar and M. B. Wise, Heavy Quark Physics (Cambridge University Press, Cambridge, England, 2000).

[73] C. Patrignani et al. (Particle Data Group), Chin. Phys. C 40, 100001 (2016). 